\title{
Precise wavefront correction with an unbalanced nulling interferometer for exo-planet imaging coronagraphs
}

\author{
J. Nishikawa ${ }^{1,2}$, L. Abe ${ }^{3,2, \star}$, N. Murakami ${ }^{1}$, and T. Kotani ${ }^{4, \star \star}$ \\ 1 MIRA project, National Astronomical Observatory of Japan, Mitaka, Tokyo 181-8588, Japan \\ e-mail: [jun.nishikawa; naoshi.murakami]@nao.ac.jp \\ 2 Extrasolar Planet Project Office, National Astronomical Observatory of Japan, Mitaka, Tokyo 181-8588, Japan \\ 3 Division of Optical and Infrared Astronomy, National Astronomical Observatory of Japan, Mitaka, Tokyo 181-8588, Japan \\ 4 Max-Planck-Institut für Radioastronomie, Auf dem Hügel 69, 53121 Bonn, Germany
}

Received 22 May 2006 / Accepted 3 June 2008

\section{ABSTRACT}

\begin{abstract}
Context. Coronagraphs of high dynamical range used for direct exo-planet detection $\left(10^{9}-10^{10}\right.$ contrast) on small angular separation (few $\lambda / D$ units) usually require an input wavefront quality of approximately ten thousandths of a wavelength rms.

Aims. We propose a novel method based on a pre-optics setup that behaves partly as a low-efficiency coronagraph, and partly as a high-sensitivity wavefront aberration compensator (phase and amplitude). The combination of the two effects results in a highly accurate corrected wavefront.

Methods. First, an (intensity-) unbalanced nulling interferometer (UNI) performs a rejection of part of the wavefront electric field. Then, the input aberrations of the recombined output wavefront are magnified. Because of the unbalanced recombination scheme, aberrations can be free of phase singular points (zeros) and can therefore be compensated by a downstream phase and amplitude correction (PAC) adaptive optics system, using two deformable mirrors.

Results. In the image plane, the central star's peak intensity and the noise level of its speckled halo are reduced by the UNI-PAC combination: the output-corrected wavefront aberrations can be interpreted as an improved compensation of the initial (eventually already corrected) incident wavefront aberrations.

Conclusions. The important conclusion is that not all of the elements in the optical setup using UNI-PAC need to reach the $\lambda / 10000$ rms surface error quality.
\end{abstract}

Key words. instrumentation: interferometers - instrumentation: adaptive optics - techniques: interferometric - planetary systems

\section{Introduction}

Optical coronagraphy in space is one of the most useful methods to achieve high dynamic range observations for the direct detection of extra-solar planets (e.g. Coulter 2005; Aime \& Vakili 2006). A coronagraph can reduce a central star intensity and its diffracted halo light around the star where exo-planets would appear. Several coronagraph designs using advanced focal plane masks (Guyon et al. 1999; Kuchner \& Traub 2002; Baba \& Murakami 2002; Riaud et al. 2003) were reported, which reduce the light energy, sometimes to zero in theory, inside a re-imaged pupil plane called a Lyot stop, and also at a final image. Some techniques of nulling interferometry, which have been considered mainly for mid-infrared long-baseline interferometers (Mennesson et al. 2005), have been applied to the operations of optical coronagraphs in a single telescope using rotation-shearing interferometers (Baudoz et al. 2000; Tavrov et al. 2005) or lateral-shearing interferometers with overlapped or separated sub-apertures (Shao et al. 2004;

\footnotetext{
* Current address: Laboratoire Hippolyte Fizeau, UMR 6525 Université de Nice-Sophia Antipolis, Parc Valrose, 06108 Nice, France; e-mail: Lyu.Abe@unice.fr

$\star \star$ Current address: LESIA, Observatoire de Paris, section Meudon, 5 place Jules Janssen, 92195 Meudon, France;

e-mail: takayuki.kotani@obspm.fr
}

Nishikawa et al. 2005). Pupil function modification can reduce only the halo intensity by using shaped or apodized pupils (Kasdin et al. 2005; Galicher et al. 2005). Wavefront phase control by an adaptive optics (AO) system can reduce the halo intensity of a limited area, called "dark hole" (Malbet et al. 1995). Multiple-stage coronagraphs (Aime \& Soummer 2004; Tolls et al. 2005), and combinations of these methods, or pre-optics schemes have been proposed to achieve a higher dynamic range (Nishikawa et al. 2005; Nishikawa \& Murakami 2006; Abe et al. 2006).

In any case, the required dynamic range of $10^{9}-10^{10}$ for direct detection of earth-like planets by optical coronagraphs can be achieved only with a very high quality wavefront of $\lambda / 10000 \mathrm{rms}$ and an intensity uniformity of $1 / 1000 \mathrm{rms}$ (Kuchner \& Traub 2002; Lowman et al. 2004). Wavefront aberrations throughout the optical train produce a so-called speckle halo noise in the image plane (e.g. Bordé \& Traub 2006) that prevents direct detection of the planet if these speckles are too bright. Manufacturing or polishing accurate mirrors is far more straightforward for small mirrors than large mirrors, for which it can be difficult to achieve the required surface accuracy. For large mirror telescopes, an AO system can be used to help achieve the necessary wavefront accuracy, where both measurements and corrections of the wavefront are important. Giant or young planets brighter than $10^{6}$ contrast are the target of ground-based telescopes of infrared wavelengths where 
wavefront correction by an $\mathrm{AO}$ system is also a key technology for use against atmospheric turbulence (e.g. Makidon et al. 2006; Serabyn et al. 2006; Tamura et al. 2006).

In an AO system, a deformable mirror (DM) is controlled by a signal from a wavefront sensor (WFS). Control accuracy of a DM was demonstrated to a level of $\lambda / 10000 \mathrm{rms}$ (e.g. Brown et al. 2003; Evans et al. 2005), while some commercial wavefront sensors were reported to achieve absolute wavefront measurement accuracies of $\lambda / 1000 \mathrm{rms}$ by the conventional Shack-Hartmann method (e.g. HASO HP 26 from Imagine Optic). For interferometric sensors, repeatability accuracies of approximately $\lambda / 10000 \mathrm{rms}$ were demonstrated (e.g. Zygo VeriFire AT). In the high dynamic range coronagraph regime, however, a non-common path error problem, i.e. differences between optics of the WFS and the main path for the star and the planet images, should be considered in the pupil plane WFS such as the Shack-Hartmann sensor, the interferometer, or a curvature sensor (e.g. Guyon 2006).

Solutions for the non-common path error problem are focal plane WFSs (Codona \& Angel 2004; Bordé \& Traub 2006; Give' on et al. 2006) or speckle nulling, which feature focal plane speckle measurement and iterative wavefront control. A dynamic range of $10^{9}$ within the dark hole was achieved after a few thousand iterations by an AO system (Balasubramanian et al. 2006), where the wavefront correction approaches the requirement.

These focal-plane sensing approaches, however, meet other problems. When the speckle intensity level becomes closer to the planet intensity, long exposure times are required to obtain higher quality $\mathrm{S} / \mathrm{N}$ measurements of the residual wavefront aberrations. In addition, the wavefront stability of the entire telescope system must be guaranteed during this time lapse. A multiplestage coronagraph that reduces speckles with AO at every stage (Tolls et al. 2005) faces the same problem. In the case of multiple stages for approaches using nulling coronagraphs or nulling interferometers, no wavefront sensing was previously considered (e.g. Nishikawa et al. 2005; Nishikawa \& Murakami 2006). However, the same wavefront sensing problems would exist, i.e. the intensity in the pupil plane (at the Lyot stop) could be zero or low, exhibiting a random intensity pattern where phase singularity at zero intensity points makes wavefront measurements and corrections difficult. For high dynamic range optics designed to detect exo-planets directly, maintaining wavefront quality and the development of coronagraphs are important issues.

We propose a pre-optics scheme for coronagraphs, using a combination of an unbalanced nulling interferometer (UNI) and a two-DM AO system (Fig. 1) for Phase and Amplitude Correction (PAC). Similarities can be found with previous studies, i.e. pre-optics by a balanced nulling interferometer (Nishikawa et al. 2005; Nishikawa \& Murakami 2006), coronagraphic pre-optics with non-zero amplitude distribution in the pupil after an interference and complex amplitude correction (Abe et al. 2006), and a multiple-stage wavefront correction by commercial quality AO systems (Tolls et al. 2005).

The pre-optics concept using a balanced nulling interferometer in front of other coronagraphs was shown to be effective in absorbing part of the required dynamic range (Nishikawa et al. 2005; Nishikawa \& Murakami 2006) but could not maintain sufficiently high quality wavefront after the interference to be corrected again by a secondary AO system. As a consequence, it places strong constraints on the precision and accuracy of the upstream AO system. Abe et al. (2006) showed that pre-optics that use a pseudo-coronagraphic stage and a complex amplitude filter at the Lyot plane can produce

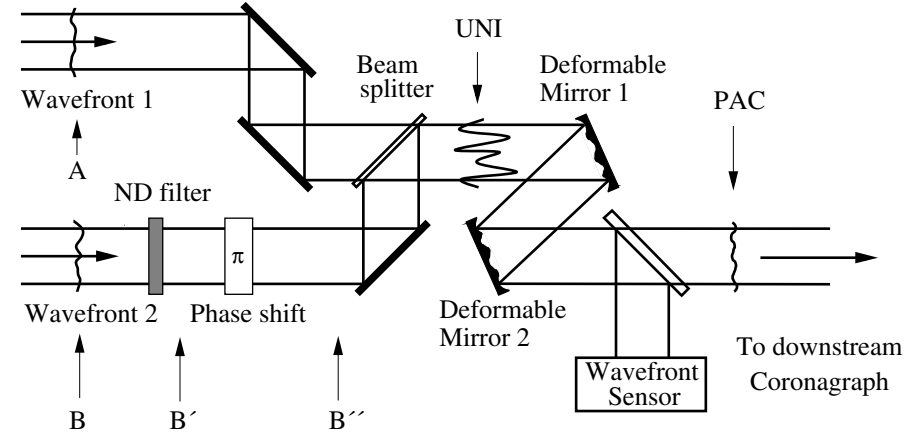

Fig. 1. Schematic of a possible optical layout for the UNI-PAC method (unbalanced nulling interferometer followed by a phase and amplitude correction adaptive optics). The various optical planes are those referred to in the text.

a flat and almost uniform wavefront, in spite of central obscuration and spider-arm patterns (i.e. large amplitude aberrations at the entrance pupil). We extended this concept to facilitate the phase and amplitude measurement/correction after a nulling stage, which is usually the bottleneck of high contrast coronagraphs. In the proposed concept, the UNI attempts simultaneously to perform the absorption of part of the dynamic range, and the magnification of wavefront aberrations without phase singularities for later compensation by an AO system (i.e. the PAC), so that the star's speckled halo is attenuated.

This paper introduces the principle of the UNI-PAC concept, a novel method that avoids the non-common path error problem and the low intensity or phase singularity problem. The overview, formalization, and simulation of the concept are addressed in Sects. 2-4, respectively, followed by a discussion in Sect. 5. Conclusions are drawn in Sect. 6.

\section{Overview of the concept}

A description of the UNI-PAC process is as follows. Figure 1 shows a possible optical layout of the proposed concept, the UNI-PAC method, and Fig. 2 depicts the associated effects on the amplitude and phase in the pupil plane at different points in the set-up. The UNI uses two wavefronts extracted from a collimated beam of a telescope. Aberrations in the collimated beam can be compensated by an AO system, for instance, at a reference level of $\lambda / 1000 \mathrm{rms}$ that we use throughout this paper.

In our optical scheme, the UNI stage is intended to produce two effects. First, it reduces the intensity of the star image by, for example, approximately $1 / 100$, but has no effect on the speckle halo level. This corresponds to an amplitude reduction in the unaberrated electric field by a partial nulling in the pupil plane.

A second effect of the UNI is the wavefront aberration magnification (for instance, from $\lambda / 1000$ to $\lambda / 100$ ), which is a consequence of the partial nulling and one of the most important phenomenon in the UNI-PAC concept. It is consistent with the image plane observation that the difference between the speckle level and the attenuated star intensity becomes smaller.

The next stage is the PAC, that is, a second AO system whose goal is to compensate for the magnified phase and amplitude aberrations (from $\lambda / 100$ to $\lambda / 1000$ in our example). The corresponding effect in the image plane is the reduction in the speckled halo level. The PAC stage can control the wavefront because the UNI stage has maintained an almost uniform intensity level in the pupil plane (i.e. with no phase singularities).

In our example, before the UNI stage, the initial intensity ratios of the star's peak intensity, the speckle halo, and the 

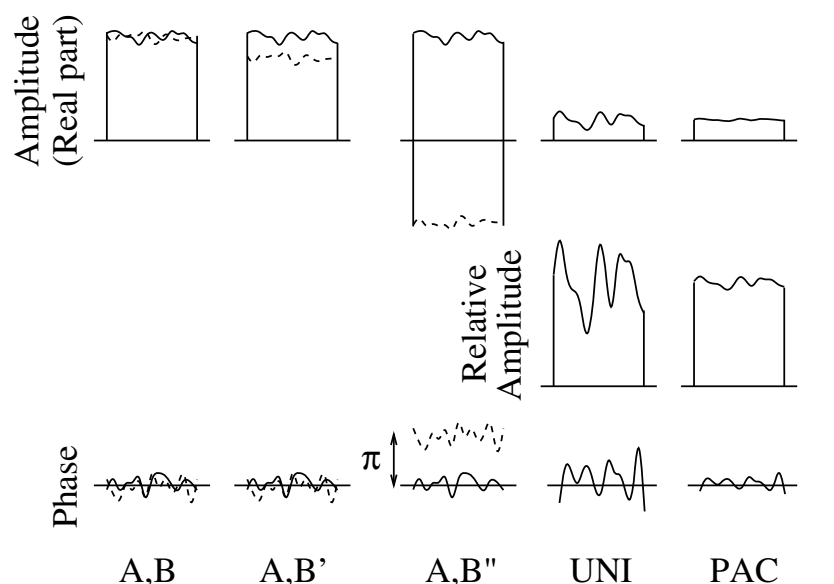

Fig. 2. Schematic of wavefront phase and amplitude magnification in the process of the UNI-PAC method, where only the real part is indicated for the amplitude. In planes A), B), B'), B') solid and dashed lines show wavefront 1 and 2, respectively. In planes UNI and PAC the combined wavefront is shown.

planet are $1: 10^{-7}: 10^{-9}$ under a wavefront aberration of about $\lambda / 1000 \mathrm{rms}$. After the UNI, the ratios become $10^{-2}: 10^{-7}: 10^{-9}$. Here the starlight is reduced but the speckle level remains unchanged. After the PAC stage, the speckle is reduced and then they become $10^{-2}: 10^{-9}: 10^{-9}$. Thus, the UNI-PAC has reduced both the star and its speckled halo intensity levels by a factor of 100 .

A downstream coronagraph can then be used to decrease the star intensity further to detect the planet. We note that the coronagraph does not require $\lambda / 10000$ optics quality, but only about $\lambda / 1000$, which is sufficient to perform a $10^{-7}$ starlight attenuation, and retain the speckle level to the planet level.

We recall that the aberration level was $\lambda / 1000$, both at the UNI-PAC and the first AO system locations (which can also include the non-common path errors of the AO systems). Despite an overall optical quality of $\lambda / 1000$, we achieved a dynamic range of $10^{9}$, implying that the wavefront quality was "virtually" improved beyond the AO system capabilities. A significant advantage of using the UNI-PAC is the relaxation of the specification for the entire optics of the coronagraph, which is an important step towards developing a planet detection system in a cost-effective way.

\section{Formalism}

\subsection{Definitions and preparations}

We consider the complex amplitude of an electric field for each point in the pupil plane whose coordinate is $(x, y)$ defined by the sum of an unaberrated constant amplitude $E^{0}$ and a complex aberration term $\varepsilon$ gathering both amplitude and phase aberrations,

$E(x, y)=E^{0}+\varepsilon(x, y)$.

We prefer to use this expression instead of classical $E=E^{0}(1+\alpha) \mathrm{e}^{\mathrm{i} \theta}$ notation, where $\alpha$ and $\theta$ are the amplitude and the phase aberrations, respectively. The ratio $\varepsilon(x, y) / E^{0}$, "the normalized complex aberration", is a useful indicator of the wavefront quality in our algebraic derivations. Under small wavefront aberration conditions, its real and imaginary parts are almost equal to $\alpha(x, y)$ and $\theta(x, y)$, respectively (the correspondence between these notations can be found in Appendix A). In that way, we can treat "the normalized complex aberration" as the wavefront aberration. We assume that $E^{0}$ is a real number and that under a very small wavefront aberration condition

$\left\{\begin{aligned} E^{0} & =\langle E(x, y)\rangle \\ \langle\varepsilon(x, y)\rangle & =0,\end{aligned}\right.$

where \langle\rangle is a spatial ensemble average within the pupil plane.

The notations for the intensity of each complex amplitude of electric field that we use are

$\begin{cases}I^{0} & =\left|E^{0}\right|^{2} \\ I^{\varepsilon}(x, y) & =|\varepsilon(x, y)|^{2},\end{cases}$

where $\mid$ is the absolute value for real numbers or the modulus for complex numbers. We measure $I^{\varepsilon}$ to be the residual intensity in the pupil plane when the unaberrated component is sufficiently attenuated. The average of the residual intensity is proportional to the average speckle background intensity in the image plane.

In the following equations, the notations described above includes a suffix, indicating the location at which the wavefront is evaluated, that is, one of six planes (A, B, B', B", UNI and then PAC planes) in the optical layout of the concept of Fig. 1. In Fig. 3, a vector representation of the complex amplitude is shown for each plane in the UNI-PAC.

We consider two wavefronts $E_{\mathrm{A}}(x, y)$ and $E_{\mathrm{B}}(x, y)$ at the input of our system generated from a collimated beam of a single telescope. These wavefronts are corrected in advance by an AO system before the UNI stage, but aberrations (including noncommon path errors) remain, which we annotate with

$\left\{\begin{array}{l}E_{\mathrm{A}}(x, y)=E_{\mathrm{A}}^{0}+\varepsilon_{\mathrm{A}}(x, y) \\ E_{\mathrm{B}}(x, y)=E_{\mathrm{B}}^{0}+\varepsilon_{\mathrm{B}}(x, y) .\end{array}\right.$

Any AO system has a limited number of actuators, which limits the correction to lower spatial frequency ranges. This is a common situation in AO systems, but fortunately the low spatial frequency region corresponds to the relevant inner area in the image plane where the exo-planets would be observed. In this paper, we assume that a rather ordinary quality of $\lambda / 1000 \mathrm{rms}$ level for the AO system is used on the telescope side before the UNI-PAC optics, and another AO of the same quality is again adopted in the latter half of the optics (the PAC stage). We also consider how the combination of the unbalanced nulling interferometer and the AO system operates for this limited inner region.

For the two wavefronts, we assume the same unaberrated amplitude,

$\left\{\begin{array}{l}E_{\mathrm{A}}^{0}=E_{\mathrm{B}}^{0} \\ I_{\mathrm{A}}^{0}=I_{\mathrm{B}}^{0},\end{array}\right.$

and complex aberrations of similar level (variance) as

$\left\langle I_{\mathrm{A}}^{\varepsilon}(x, y)\right\rangle=\left\langle I_{\mathrm{B}}^{\varepsilon}(x, y)\right\rangle$.

\subsection{Unbalanced nulling interferometer (UNI) process}

The first effect of the UNI stage is a starlight attenuation by a nulling interference of the two beams. For the wavefront $E_{\mathrm{B}}$ (plane B), the modulus of the complex amplitude is slightly reduced, for instance by an $N D$ filter. At the output of this $N D$ filter, the complex amplitude becomes,

$E_{\mathrm{B}^{\prime}}(x, y)=(1-g)\left(E_{\mathrm{B}}^{0}+\varepsilon_{\mathrm{B}}(x, y)\right)$ 

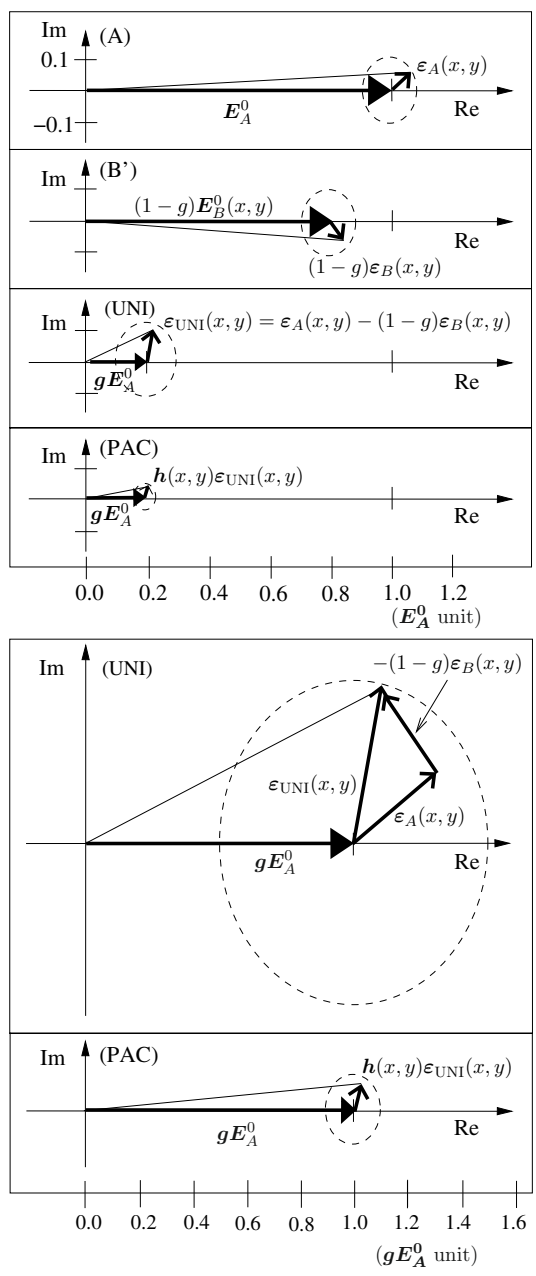

Fig. 3. Complex amplitude at a point $(x, y)$ in the pupil plane under the processes of the UNI-PAC method. In each panel, an arrow from the origin indicates the unaberrated amplitude, $E^{0}$, and the complex aberration component, $\varepsilon$, is shown by other small arrow(s), where the distribution area of the complex amplitude is drawn by a dashed-lined ellipse. Traditional phase aberration, $\theta$, is indicated by an arc. The upper four panels indicate the complex amplitude of beam A, beam B with $N D$ filter before $\pi$ phase shift, UNI, and finally PAC planes respectively from top to bottom, in $E_{\mathrm{A}}^{0}$ units. The two lower panels are similar to the last two frames of the upper panel, except that the vectors have been normalized by the factor $g E_{\mathrm{A}}^{0}$, where $g=0.2$. The normalized aberration is magnified by $1 / g$ by the UNI (UNI output plane), and compensated by a factor $h(x, y)$ at the PAC output plane.

where $g$ is a small fraction of unity, i.e. the unbalance factor. The beam is then phase-shifted, such that

$$
\begin{aligned}
E_{\mathrm{B}^{\prime \prime}}(x, y) & =\mathrm{e}^{\mathrm{i} \phi}(1-g)\left(E_{\mathrm{B}}^{0}+\varepsilon_{\mathrm{B}}(x, y)\right) \\
& =-(1-g)\left(E_{\mathrm{B}}^{0}+\varepsilon_{\mathrm{B}}(x, y)\right),
\end{aligned}
$$

where we considered $\phi=\pi$.

The two wavefronts $E_{\mathrm{A}}(x, y)$ and $E_{\mathrm{B}^{\prime \prime}}(x, y)$ are summed for destructive interference, so that at the UNI output plane (subscripted UNI), and using Eq. (5),

$E_{\mathrm{UNI}}(x, y)=g E_{\mathrm{A}}^{0}+\varepsilon_{\mathrm{A}}(x, y)-(1-g) \varepsilon_{\mathrm{B}}(x, y)$,

where we omitted the reflection and transmission ratio of the beam combiner.

There is one point that we need to clarify: the important effect we want to achieve is to obtain an appropriate value for the modulus of the unaberrated component of the residual complex amplitude after the beam combination. In other words, it does not matter whether this residual is purely real or not. Therefore, in Eq. (8), the factor $\mathrm{e}^{\mathrm{i} \phi}(1-g)$ can be complex, as long as we can control either $g$ or $\phi$ (or both) to produce the desired unaberrated modulus amplitude for the combined beam. In the following, we assume $\phi=\pi$ for simplicity.

We consider that defects in the optics for the unbalancing and the phase shift are negligible, or included in $\varepsilon_{\mathrm{B}}$. The aberrations of the beam combiner and the two optical paths in the UNI are treated in the same way using $\varepsilon_{\mathrm{A}}$ and $\varepsilon_{\mathrm{B}}$.

The unaberrated complex amplitude of Eq. (9) and its intensity become

$$
\left\{\begin{array}{l}
E_{\mathrm{UNI}}^{0}=g E_{\mathrm{A}}^{0} \\
I_{\mathrm{UNI}}^{0}=g^{2} I_{\mathrm{A}}^{0} .
\end{array}\right.
$$

At this point, the starlight intensity is reduced by $g^{2}$, for instance, if $g=0.1$ the starlight is attenuated by $1 / 100$. The complex aberration term is almost the sum of the two wavefronts as

$$
\left\{\begin{array}{l}
\varepsilon_{\mathrm{UNI}}(x, y)=\varepsilon_{\mathrm{A}}(x, y)-(1-g) \varepsilon_{\mathrm{B}}(x, y) \\
\left\langle I_{\mathrm{UNI}}^{\varepsilon}(x, y)\right\rangle=\left(2-2 g+g^{2}\right)\left\langle I_{\mathrm{A}}^{\varepsilon}(x, y)\right\rangle,
\end{array}\right.
$$

where Eq. (6) and the independence of $\varepsilon_{\mathrm{A}}(x, y)$ and $\varepsilon_{\mathrm{B}}(x, y)$ are used for the latter equation. This situation corresponds to a "worst case" because if the aberrations are perfectly correlated so that $\varepsilon_{\mathrm{A}}(x, y)=\varepsilon_{\mathrm{B}}(x, y)$, then $I_{\mathrm{UNI}}^{\varepsilon}(x, y)=g^{2} I_{\mathrm{A}}^{\varepsilon}(x, y)$, implying that the aberration term in addition to the unaberrated amplitude is extinguished. Therefore, a more accurate result than that provided by Eq. (11) may be achievable if the wavefronts were partially correlated (see Appendix B). We continue with the worst case formula, which provides that the UNI-PAC process works well.

To evaluate the dynamic range improvement achieved by the UNI stage, the unaberrated and the aberrated component of the star should be compared with the planet. We introduce the intensity of the planet $I_{\mathrm{A}}^{\mathrm{p}}$, where we consider only the unaberrated component, since its aberration component intensity is small. We assume that the planet intensity after the UNI is given by

$I_{\mathrm{UNI}}^{\mathrm{p}}=\left(2-2 g+g^{2}\right) I_{\mathrm{A}}^{\mathrm{p}}$

by averaging over the constructive and the destructive conditions of the interferometer (see Appendix C). Using Eqs. (10), (11), and (12), the dynamic range improvement through the UNI stage is then expressed as

$\left\{\begin{array}{c}\frac{I_{\mathrm{UNI}}^{0}}{I_{\mathrm{UNI}}^{\mathrm{p}}}=\frac{g^{2}}{2-2 g+g^{2}} \frac{I_{\mathrm{A}}^{0}}{I_{\mathrm{A}}^{\mathrm{p}}} \\ \frac{\left\langle I_{\mathrm{UNI}}^{\varepsilon}(x, y)\right\rangle}{I_{\mathrm{UNI}}^{\mathrm{p}}}=\frac{\left\langle I_{\mathrm{A}}^{\varepsilon}(x, y)\right\rangle}{I_{\mathrm{A}}^{\mathrm{p}}},\end{array}\right.$

where the latter equation shows that the averaged aberration intensity is unaffected by the UNI, while the star intensity becomes closer to the planet intensity by a factor of $g^{2} /\left(2-2 g+g^{2}\right)$.

\subsection{Wavefront aberration magnification by UNI stage}

The important phenomenon at the UNI stage that we must emphasize is the wavefront aberration magnification. The 
normalized complex aberration after the UNI stage can be evaluated, using Eqs. (10) and (11), to be,

$\left\{\begin{array}{c}\frac{\varepsilon_{\mathrm{UNI}}(x, y)}{E_{\mathrm{UNI}}^{0}}=\frac{1}{g} \frac{\varepsilon_{\mathrm{A}}(x, y)-(1-g) \varepsilon_{\mathrm{B}}(x, y)}{E_{\mathrm{A}}^{0}} \\ \left\langle\left|\frac{\varepsilon_{\mathrm{UNI}}(x, y)}{E_{\mathrm{UNI}}^{0}}\right|^{2}\right\rangle=\frac{2-2 g+g^{2}}{g^{2}}\left\langle\left|\frac{\varepsilon_{\mathrm{A}}(x, y)}{E_{\mathrm{A}}^{0}}\right|^{2}\right\rangle,\end{array}\right.$

where the UNI magnifies the normalized complex aberration by a factor $1 / g$ (recalling that $g<1$ ) compared to the initial wavefront aberrations, or, in average by $\sqrt{2-2 g+g^{2}} / g$.

To obtain an appropriate magnification of the wavefront aberration, we chose a sufficiently large value of the unbalance factor $g$, so that $E_{\mathrm{UNI}}(x, y)$ always has a positive intensity (modulus) without any phase singularities, $\left|E_{\mathrm{UNI}}(x, y)\right|>0$. A little tighter constraint is also suitable,

$\left|E_{\mathrm{UNI}}^{0}\right|>\left|\varepsilon_{\mathrm{UNI}}(x, y)\right|$,

which implies that $E_{\mathrm{UNI}}(x, y)$ is distributed over half of the complex plane excluding the origin (see Fig. 3). According to the conditions of this equation, the smaller is the value of $g$, the greater is the extent to which both the star signal is attenuated and the aberration is magnified.

An example of the aberration magnification by the UNI stage is shown in Fig. 4, where one wavefront has a phase aberration of $\lambda / 1000 \mathrm{rms}$, an amplitude aberration of $0.004 \mathrm{rms}$, and an unbalance factor $g=0.1$. The initial aberration is $\left|\varepsilon_{\mathrm{A}}(x, y) / E_{\mathrm{A}}^{0}\right|<0.02$ and the magnified one is $\left|\varepsilon_{\mathrm{UNI}}(x, y) / E_{\mathrm{UNI}}^{0}\right|<0.2$, which is decreased to lower than 0.02 by the PAC stage. Although $g=0.02$ can be adopted to satisfy Eq. (15), a magnified wavefront aberration close to unity $\left|\varepsilon_{\mathrm{UNI}}(x, y) / E_{\mathrm{UNI}}^{0}\right| \sim 1$ would be less favorable in performing the wavefront correction at the PAC stage.

If $g \rightarrow 0$, the unaberrated amplitude becomes negligible, $E_{\mathrm{UNI}}^{0} \rightarrow 0$, and the nulling result consists of only the aberration terms $E_{\mathrm{UNI}}(x, y)=\varepsilon_{\mathrm{A}}(x, y)-\varepsilon_{\mathrm{B}}(x, y)$, which are distributed around the origin in the complex plane. In this case, the residual intensity in the pupil plane takes the form of a speckled field with a small inhomogeneous amplitude and random phase fluctuations, resulting in the existence of zero amplitude points. Consequently, low light levels and zero intensity points with phase singularities make it difficult to measure and correct the wavefront with an AO system. This is exactly the problem situation that we aim to avoid with the unbalanced nulling.

\subsection{Phase and amplitude correction (PAC) process}

After the wavefront aberrations are magnified by the UNI stage, they must be reduced to a level equivalent to $\varepsilon_{\mathrm{A}}(x, y) / E_{\mathrm{A}}^{0}$ (the initial wavefront aberration) by a secondary AO system. To perform the phase and amplitude correction (i.e., the PAC), the UNI is followed by a second AO system composed of two DMs (Fig. 1) and a WFS that measures the wavefront shape and the intensity distribution. The compensation level can be set differently according to the characteristics of each AO system.

The PAC process is formalized by applying a wavefrontaberration-reduction function $h(x, y)$ to the normalized complex aberration,

$$
\left\{\begin{array}{c}
\frac{\varepsilon_{\mathrm{PAC}}(x, y)}{E_{\mathrm{PAC}}^{0}}=h(x, y) \frac{\varepsilon_{\mathrm{UNI}}(x, y)}{E_{\mathrm{UNI}}^{0}} \\
\left\langle\left|\frac{\varepsilon_{\mathrm{PAC}}(x, y)}{E_{\mathrm{PAC}}^{0}}\right|^{2}\right\rangle=k^{2}\left\langle\left|\frac{\varepsilon_{\mathrm{UNI}}(x, y)}{E_{\mathrm{UNI}}^{0}}\right|^{2}\right\rangle
\end{array}\right.
$$

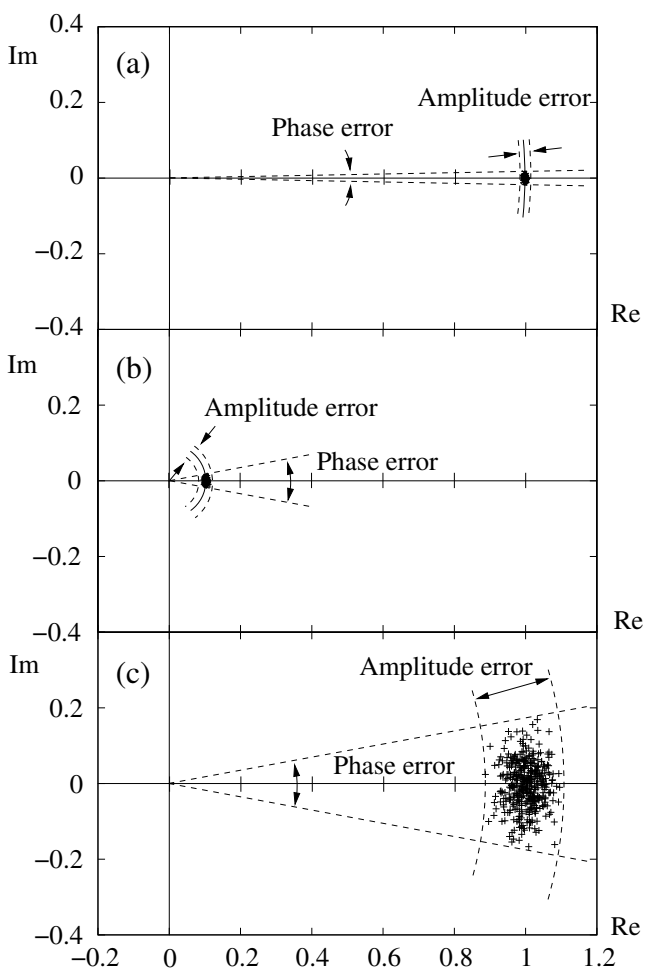

Fig. 4. Wavefront aberration magnification by the unbalanced nulling interference. a) Complex amplitude of 400 points is simulated in a wavefront whose phase aberration rms is 0.0063 radian and amplitude aberration rms is 0.004 . b) After the unbalanced nulling interference with a no-aberration wavefront with an amplitude ratio of 0.9 , the phase aberration is easily seen as magnified, and c) the amplitude aberration magnification is also obvious when the complex numbers are normalized by the unaberrated wavefront electric field, which is $0.1 E^{0}$ in this case if we represent the original unaberrated field as $E^{0}$. The magnified aberrations seen in panel c) can be corrected again to the same quality as a) by an $\mathrm{AO}$ system in the next stage.

where $k$ is a kind of weighted average of the aberration-reduction function $h(x, y)$ defined to be

$k^{2}=\frac{\left\langle\left|h(x, y) \varepsilon_{\mathrm{UNI}}(x, y)\right|^{2}\right\rangle}{\left\langle\left|\varepsilon_{\mathrm{UNI}}(x, y)\right|^{2}\right\rangle}$.

Since the residual aberrations before and after the UNI-PAC are caused by sensing, control, specifications of the DMs, or noncommon path errors of each AO system, then $\varepsilon_{\mathrm{PAC}}(x, y)$ or $h(x, y)$ is not correlated with $\varepsilon_{\mathrm{UNI}}(x, y)$.

After the PAC operation, the unaberrated complex amplitude can be

$\left\{\begin{array}{l}E_{\mathrm{PAC}}^{0}=E_{\mathrm{UNI}}^{0} \\ I_{\mathrm{PAC}}^{0}=I_{\mathrm{UNI}}^{0}\end{array}\right.$

In the equations above, we assumed a negligible intensity change due to the amplitude control of the PAC DMs (under small wavefront aberration conditions). If we consider this change in intensity, we need to introduce a parameter $\xi$ such that $E_{\mathrm{PAC}}^{0}=\xi E_{\mathrm{UNI}}^{0}$ with $\xi \sim 1$ in our equations. However, its effect on the principle of the present concept should not be large. In the following, we do not take this effect into consideration.

In the PAC output plane, the complex aberration term becomes

$\left\{\begin{array}{l}\varepsilon_{\mathrm{PAC}}(x, y)=h(x, y) \varepsilon_{\mathrm{UNI}}(x, y) \\ \left\langle I_{\mathrm{PAC}}^{\varepsilon}(x, y)\right\rangle=k^{2}\left\langle I_{\mathrm{UNI}}^{\varepsilon}(x, y)\right\rangle .\end{array}\right.$ 
In total the complex amplitude is

$E_{\mathrm{PAC}}(x, y)=g E_{\mathrm{A}}^{0}+h(x, y)\left(\varepsilon_{\mathrm{A}}(x, y)-(1-g) \varepsilon_{\mathrm{B}}(x, y)\right)$.

For the planet, the intensity change caused by the PAC stage can be considered negligible for reasons similar to that for Eq. (18),

$I_{\mathrm{PAC}}^{\mathrm{p}}=I_{\mathrm{UNI}}^{\mathrm{p}}$.

The improvement in the dynamic range at the PAC stage is then just the aberration component reduction expressed as,

$\left\{\begin{array}{c}\frac{I_{\mathrm{PAC}}^{0}}{I_{\mathrm{PAC}}^{\mathrm{p}}}=\frac{I_{\mathrm{UNI}}^{0}}{I_{\mathrm{UNI}}^{\mathrm{p}}} \\ \frac{\left\langle I_{\mathrm{PAC}}^{\varepsilon}(x, y)\right\rangle}{I_{\mathrm{PAC}}^{\mathrm{p}}}=k^{2} \frac{\left\langle I_{\mathrm{UNI}}^{\varepsilon}(x, y)\right\rangle}{I_{\mathrm{UNI}}^{\mathrm{p}}} .\end{array}\right.$

If we want to express the PAC stage performance compared to the initial AO-compensated wavefront level, we can write,

$\left\langle\left|\frac{\varepsilon_{\mathrm{PAC}}(x, y)}{E_{\mathrm{PAC}}^{0}}\right|^{2}\right\rangle=\eta^{2}\left\langle\left|\frac{\varepsilon_{\mathrm{A}}(x, y)}{E_{\mathrm{A}}^{0}}\right|^{2}\right\rangle$,

where $\eta \sim 1$ means that the residual wavefront aberration after the PAC is similar to that delivered by the first AO system. A smaller value of $\eta$ would imply a more effective compensation by the PAC stage, and inversely a larger value should correspond to less effective compensation. Then, with Eqs. (14) and (16), we obtain

$k^{2}=\eta^{2} g^{2} /\left(2-2 g+g^{2}\right)$.

This expression shows that, when $\eta \sim 1, k^{2}$ is a number less than unity almost completely defined by the unbalance factor $g$ of the UNI stage.

\subsection{UNI-PAC total effect}

From Eqs. (13) and (22), the total reduction effect on the intensities of the unaberrated and aberrated wavefront components are respectively,

$$
\left\{\begin{array}{c}
\frac{I_{\mathrm{PAC}}^{0}}{I_{\mathrm{PAC}}^{\mathrm{P}}}=\frac{g^{2}}{2-2 g+g^{2}} \frac{I_{\mathrm{A}}^{0}}{I_{\mathrm{A}}^{\mathrm{P}}} \\
\frac{\left\langle I_{\mathrm{PAC}}^{\varepsilon}(x, y)\right\rangle}{I_{\mathrm{PAC}}^{\mathrm{P}}}=k^{2} \frac{\left\langle I_{\mathrm{A}}^{\varepsilon}(x, y)\right\rangle}{I_{\mathrm{A}}^{\mathrm{P}}} .
\end{array}\right.
$$

Equivalently in the image plane, the central star profile attenuation factor is $g^{2} /\left(2-2 g+g^{2}\right)$ and the average speckle halo attenuation factor is $k^{2}$. These results can be seen in Fig. 5, based on a simplified numerical simulation where the corrected wave after the PAC stage is calculated by generating random residual aberrations.

The important conclusion is that the sequential combination of the UNI and PAC achieves an aberration compensation of the star wavefront amplitude beyond the correction capabilities of the individual AO systems used (including the non-common path errors). We wish to call this a virtual compensation.

After these pre-optics, a downstream coronagraph should study the remainder of the total dynamic range, where the requirements for wavefront aberration level and star intensity suppression level are relaxed by the reduction factors of the unaberrated and the aberration wavefront (the star and the speckle) intensities in Eq. (25). The detailed contrast improvement in the image plane by this concept depends on the spectrum of the aberrations and the specifications of the AO system, although the (average) relative improvement in the dynamic range is the same as the pupil plane.

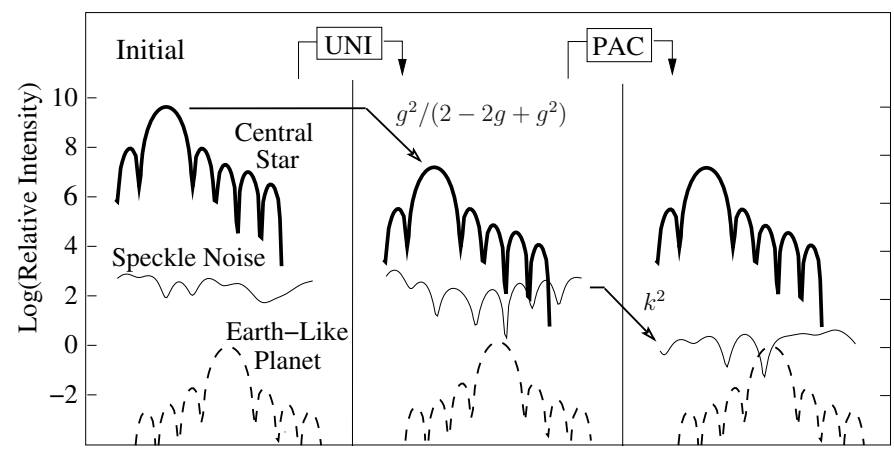

Fig. 5. Results of a simplified numerical simulation showing the improvement of the dynamic range in the image plane by the UNI-PAC method. The curves show the central star (bold line) and its speckle halo noise (solid line) normalized by the planet peak intensity (dashed line). Initially (left panel) the contrast between the star and the planet is assumed to be $5 \times 10^{9}$ and the speckle halo lies $4 \times 10^{2}$ above the planet, which is a result of the phase aberration of $\lambda / 1000 \mathrm{rms}$ and the amplitude aberration of $0.004 \mathrm{rms}$ with a flat spectrum $(30 \times 30$ square grids in the pupil). After the UNI is applied with the factor $g=0.1$ (middle panel $)$, the central star intensity is reduced by $g^{2} /\left(2-2 g+g^{2}\right)=0.0055$ times. After the PAC process (right panel), the aberration-magnified wavefront is compensated to the same aberration rms as the input. The speckle halo noise is then decreased $k^{2}=0.0055$ times, that is, twice the planet peak intensity. The star profile is drawn as a point spread function of a non-modified pupil, which can be suppressed by a downstream coronagraph.

\section{Simulation of the UNI-PAC}

We attempt to determine in a more global way how the UNI-PAC can perform, aside from purely analytical considerations. In our simulations, we use the Fourier optics approximation and FFT to produce complex fields in conjugated planes. The top row of Fig. 6 shows the distribution of the two complex amplitudes of the beams A and B. The initial phase aberrations are uniformly distributed over pupil spatial frequencies (flat power spectrum) with an rms amplitude of $\lambda / 1000$, whereas the amplitude aberrations follow a $f^{-2.5}$ power-law with an amplitude of $1 / 200$ th of the average amplitude level. Beam B is shown after passing through a neutral density filter with $81 \%$ transmission, meaning that at the UNI output plane, the recombined beam intensity is reduced by a factor $g^{2}=0.01$, and the phase aberrations are amplified. The PAC stage uses two deformable mirrors (DM1 and DM2). DM1 modulates the UNI output beam in phase only so that the curvature of the wavefront induces an amplitude modification by beam propagation to the location of DM2. Once the phase and amplitude after the UNI are measured, we use an FFT-based propagation algorithm combined with a recursive Gerchberg-Saxton (Gerchberg \& Saxton 1972, and e.g. Fienup 1982) algorithm, to find the DM1 phase that converges toward a given pupil amplitude distribution (flat in this case). This step is shown in the middle row of Fig. 6. The left panel shows the initial propagated beam amplitude, and the right panel shows the same beam, but after convergence of the algorithm. DM2 compensates for the phase aberrations including those at the UNI output and the effects of phase modulation by DM1. We did not use a realistic phase analysis/correction scheme. We assumed that the sensing sensitivity was sufficiently high to provide a relative phase quality similar to that used for the input beams (Beam A and Beam B). In this simulation, the DMs were $32 \times 32$ actuators (793 within a circular pupil are effective over a total of 1024). The distance between the two DMs in the PAC stage was set to a relatively arbitrary distance of $30 \mathrm{~cm}$ 

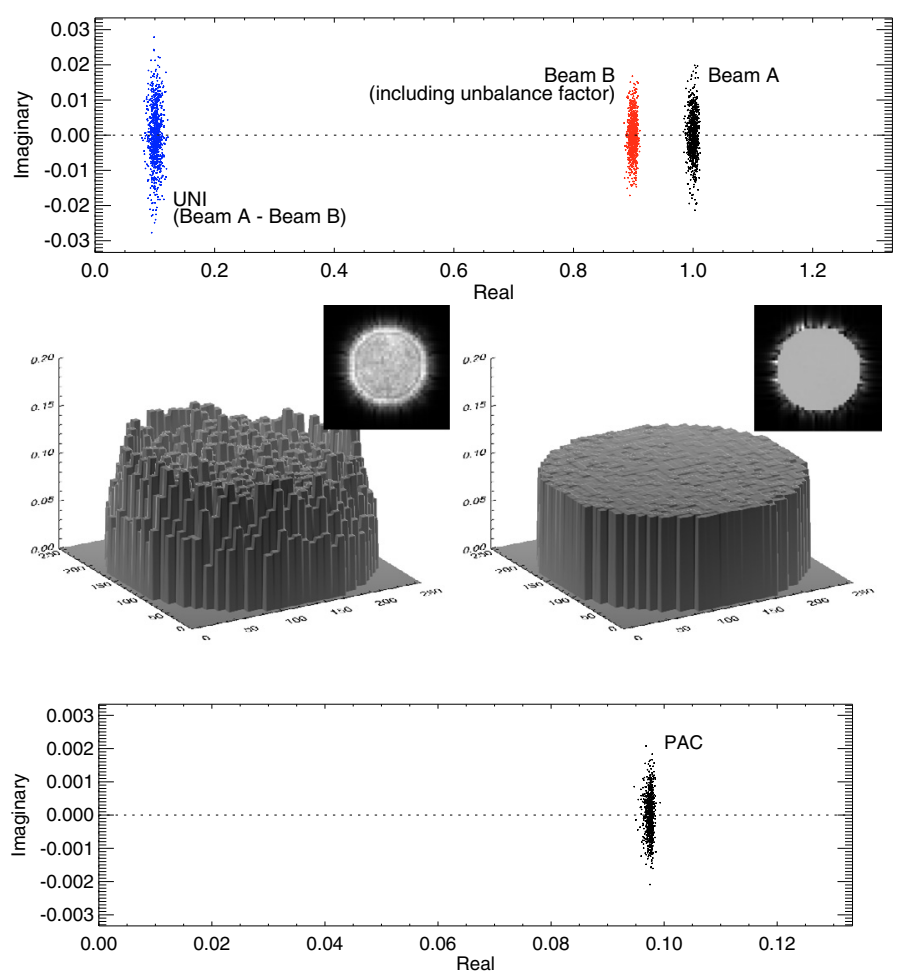

Fig. 6. Simulation of the UNI-PAC resulting in a $\sim \lambda / 10000 \mathrm{rms}$ from input wavefronts quality of $\sim \lambda / 1000 \mathrm{rms}$. The top row shows the complex amplitude distribution in the complex plane for the input Beam A, Beam B (including the unbalance factor $g=0.1$ ), and for the unbalanced combined beam, i.e. at the UNI output plane. The middle row shows the amplitude (modulus) distribution of UNI output beam after the propagation between DM1 and DM2, before (left), and after (right) the Gerchberg-Saxton algorithm convergence. DM1 compensates for amplitude fluctuations only, while DM2 compensates for the phase. The bottom row shows the PAC output wavefront complex distribution. Note that the real and imaginary axes scale has been reduced by a factor of $1 / 10$.

(propagation distance), while the collimated beam diameter was $1 \mathrm{~cm}$. These parameters certainly need to be adjusted, especially with respect to the DMs dynamical range and the actuators influence functions, but this is not the purpose of this paper. The bottom of Fig. 6 shows the final corrected wavefront at the PAC output plane. It has approximately the same wavefront aberration quality as the input beams, both in phase and amplitude.

Figure 7 shows various plots corresponding to different steps of this UNI-PAC simulation. The top dotted line is the profile of the PSF computed from Beam A. For comparison purposes, the corresponding thick dotted curve (speckle halo around $10^{7}$ contrast level) is the profile of an image given by an "ideal" coronagraph (which perfectly cancels the unaberrated plane wave) with this input wavefront. The dashed and continuous thinner curves are the image profiles at the UNI output and after the PAC correction, respectively. We note the small improvement of the PAC profile (continuous line) further away in the halo, which corresponds to a more accurate wavefront. Then, we applied the same "ideal" coronagraph to the PAC output, which is shown as the thick black continuous curve at the bottom of the figure where the virtual aberration correction improvement becomes obvious. For comparison, we generated a similar coronagraphic image, but from the Beam A data of the initial wavefront, that had aberrations artificially attenuated by a factor of $1 / 10$, i.e. a wavefront with a phase aberration of $\lambda / 10000$ and amplitude aberrations of

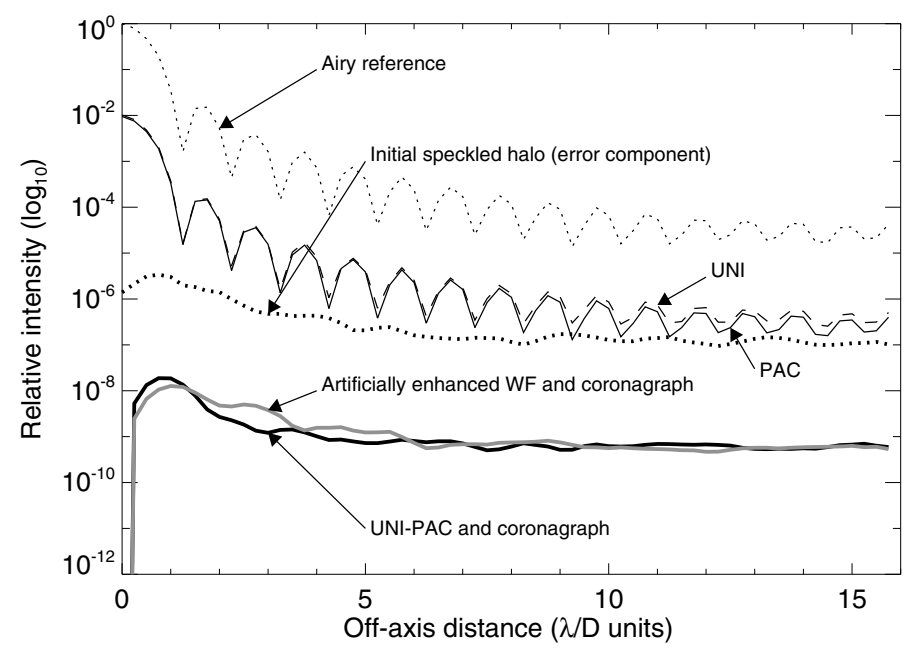

Fig. 7. Azimuthally averaged profiles of focal plane images at different locations in the UNI-PAC system: a reference Airy profile (dotted), the profile at the UNI (dashed) and the PAC (solid) planes. The thick dotted profile corresponds to the speckled halo (aberration component) before the UNI stage. The two thick lines at the bottom of this plot are (thick black) the image profile by the virtually-corrected PAC output wavefront with the ideal coronagraph, and (thick grey) the image computed by the initial wavefront with the artificial enhancement (i.e. with the complex aberrations reduced by a factor of $1 / 10$ ) combined with the ideal coronagraph.

$1 / 2000$. This latter comparison demonstrates a potential of the present method, i.e. the virtual aberration correction improvement by the UNI-PAC is equivalent to the initial compensation.

\section{Discussion}

\subsection{The virtual compensation}

In Eq. (20), at the PAC output plane, both the initial complex aberrations $\varepsilon_{\mathrm{A}}(x, y)$ and $\varepsilon_{\mathrm{B}}(x, y)$ are multiplied by a factor $h(x, y)$. This can be interpreted as the result of an unbalanced nulling interference of two wavefronts written as

$$
\left\{\begin{array}{l}
E_{\mathrm{A}}(x, y)=E_{\mathrm{A}}^{0}+h(x, y) \varepsilon_{\mathrm{A}}(x, y) \\
E_{\mathrm{B}}(x, y)=E_{\mathrm{B}}^{0}+h(x, y) \varepsilon_{\mathrm{B}}(x, y) .
\end{array}\right.
$$

In other words, it is equivalent to having a higher quality input wavefront ( $h$ times smaller aberrations), followed by an unbalanced nulling beam combination, but no further AO correction. At the UNI-PAC system output, we are unable to determine whether the input wavefront was of high quality or its quality is instead a reflection of the processing by the UNI-PAC (see also Fig. 7). We can only consider that the present concept allows for a virtual compensation beyond the AO performance.

\subsection{Chromatism issues}

The formalism in Sect. 3 is constructed for one wavelength and the method is not perfectly achromatic.

\subsubsection{Magnification process}

If the wavefront shape expressed as $z(x, y)$ (for instance, $\mathrm{nm}$ unit), is common to every wavelength because of mirror reflections, then the phase aberration $\theta(x, y)=2 \pi z(x, y) / \lambda$ is wavelength dependent. 


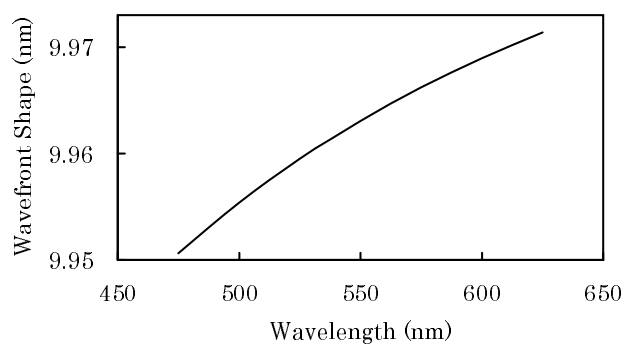

Fig. 8. Wavelength dependence of the magnified aberration. The wavefront shape aberration after the UNI, $z_{\mathrm{UNI}}$, is shown for the input wavefront shape aberration $z_{\mathrm{A}}=1 \mathrm{~nm}$ with zero amplitude aberration and $g=0.1$. In the wavelength band of $500 \mathrm{~nm}$ to $600 \mathrm{~nm}$, the wavefront aberration is magnified about 9.96 times with a small wavefront difference of about $0.01 \mathrm{~nm}$, i.e., $\lambda / 50000$ level.

For the two beams, we consider the wavefront shape aberrations of $z_{\mathrm{A}}$ (i.e. $\theta_{\mathrm{A}}=2 \pi z_{\mathrm{A}} / \lambda$ ) and $z_{\mathrm{B}}=0$ (this latter value is set to zero for convenience, but it does not affect our more general result). Wavefronts before and after the UNI are set to

$$
\left\{\begin{array}{l}
E_{\mathrm{A}}=E^{0}+\varepsilon \\
E_{\mathrm{B}}=E^{0} \\
E_{\mathrm{UNI}}=g E^{0}+\varepsilon
\end{array}\right.
$$

so that the phase aberration magnification can be written (using the notation of Appendix A and the representation of Fig. A.1)

$$
\tan \theta_{\mathrm{UNI}}=\frac{1+\varepsilon_{\mathrm{r}} / E^{0}}{g+\varepsilon_{\mathrm{r}} / E^{0}} \tan \theta_{\mathrm{A}},
$$

where $\varepsilon_{\mathrm{r}}$ is the real part of $\varepsilon$ and $\theta_{\mathrm{UNI}}$ is the phase aberration after the UNI. The aberration magnification is completed by the physical process in each wavelength and here we should check how identical the wavefront shapes $z_{\mathrm{UNI}}$ are within a wavelength band. The plot in Fig. 8 shows the wavefront aberration $z_{\mathrm{UNI}}$ at a point in the pupil plane magnified by the UNI for an initial aberration $z_{\mathrm{A}}=1 \mathrm{~nm}$ (and a zero amplitude aberration), for an unbalance factor $g=0.1$. The differential wavefront shape after the magnification is about $0.01 \mathrm{~nm}(\lambda / 50000$ level), within a wavelength band between $500 \mathrm{~nm}$ and $600 \mathrm{~nm}$. The nonlinearity of the tangent is the main cause of the wavelength dependence. The intensity-unbalanced nulling beam combiner has less dispersion, at least for an input aberration level of $\lambda / 1000$ with an aberration magnification factor of 10 .

\subsubsection{Phase shifter and ND filter}

Another issue of the achromaticity is for the $\pi$ phase shifter and the $N D$ filter in the UNI stage, i.e. a wavelength dependence of the factor $-(1-g)$ (therefore, $\phi$ and $g$ ) in Eq. (8).

In a similar way to the previous section, we consider a combination of two wavefronts by UNI written as,

$$
\left\{\begin{array}{l}
E_{\mathrm{A}}=E^{0}+\varepsilon \\
E_{\mathrm{B}}=\mathrm{e}^{\mathrm{i} \psi} E^{0} \\
E_{\mathrm{UNI}}=\left(1-(1-g) \mathrm{e}^{\mathrm{i} \psi}\right) E^{0}+\varepsilon
\end{array}\right.
$$

where $\psi$ is a (wavelength-dependent) differential phase change in the wavefront B. The aberration magnification is then expressed as

$$
\frac{\varepsilon_{\mathrm{UNI}}}{E_{\mathrm{UNI}}^{0}}=\frac{1}{1-(1-g) \mathrm{e}^{\mathrm{i} \psi}} \frac{\varepsilon}{E^{0}} .
$$

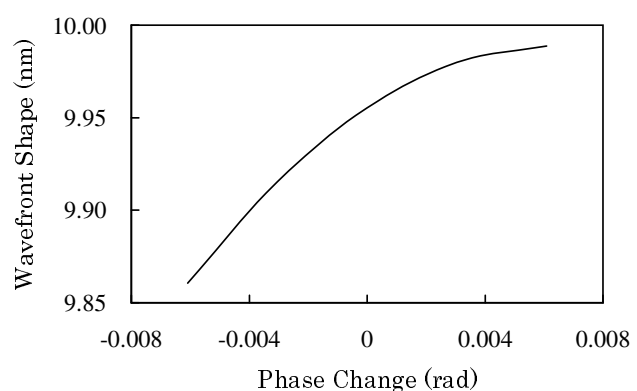

Fig. 9. Differential phase dependence of the magnified aberration. The wavefront shape aberration after the UNI, $z_{\mathrm{UNI}}$, is shown as a function of the additional phase change where the input wavefront shape aberration $z_{\mathrm{A}}=1 \mathrm{~nm}$ with zero amplitude aberration under a condition of $g=0.1$, $B=0.2 \mathrm{~m}$, and $\lambda=500 \mathrm{~nm}$. Within the phase change of $\pm 0.006 \mathrm{rad}$, the wavefront aberration is magnified about 9.95 times with a small wavefront difference of about $\pm 0.1 \mathrm{~nm}, \lambda / 5000$ level.

The magnification factor is calculated for a given differential phase change independent of its origin (such as a wavelength dependence or an incident angle to an interferometer with a baseline described in the next section). The magnified wavefront aberration through the UNI process as a function of the differential phase change is calculated and shown in Fig. 9 for the input wavefront aberration of $1 \mathrm{~nm}(\lambda / 500)$ for phase and zero for amplitude. We find that the aberration is magnified to around $9.95 \mathrm{~nm}(\lambda / 50)$ with a sufficiently small difference of about $0.1 \mathrm{~nm}(\lambda / 5000)$ within the differential phase change of $\pm 0.006 \mathrm{rad}( \pm \lambda / 1000)$. The wavelength dependence of the magnification described in the previous section is not more important than this term.

We note that for a given differential amplitude change written in a range of $g=0.1 \pm 0.001$, i.e. $\pm 0.2 \%$ difference of the transmission $\left((1-g)^{2}\right)$ of the $N D$ filter in wavelengths, a similar level of the differential aberration magnification can be found. We leave a more detailed study of these differential magnification issues to a future work.

\subsection{Resolved stellar disk}

The formalism in Sect. 3 considered an on-axis point source to be the central star. When the source has a resolved diameter, we should consider off-axis rays where the wavefront aberrations are magnified by different factors at the UNI stage. Considering the baseline B between the two interferometer apertures, the two wavefronts have an additional phase difference of $\psi=2 \pi \mathrm{B} \sin \chi / \lambda$, where $\chi$ is the incident angle of the off-axis light. We refer again to the formalism of the previous subsection to address this point.

With a baseline of $0.2 \mathrm{~m}$ (e.g. Shao et al. 2004), for instance, a 0.5 mas tilted wavefront originating in the limb position of the Sun at $10 \mathrm{pc}$ has a phase difference of $\psi=6.1 \times 10^{-3}$ radian $(\lambda / 1000)$ between the two apertures for a wavelength of $500 \mathrm{~nm}$. Therefore, the range of incident angles for the Sun at $10 \mathrm{pc}$ almost corresponds to the phase range shown by the curve in Fig. 9 with the sufficiently small differential magnification of about $\lambda / 5000$. This means that the stellar diameter might not affect the performance of a short baseline interferometer under small aberration conditions. 


\subsection{Larger phase aberrations}

Even for a large wavefront phase aberration of $\lambda / 20$ and amplitude aberration of 0.1 , the present method is still effective for the factor $g=0.3$, where the central star extinction and speckle intensity reduction becomes about 0.05 . The aberration level is similar to a ground-based AO condition and the gain of 0.05 is essential for exo-planet observations from the ground, e.g. extending the observable range from $10^{-5}$ to $5 \times 10^{-7}$. If the PAC $\mathrm{AO}$ reaches higher bandwidth than the telescope-side AO system (e.g. Guyon 2006), it might be possible to apply the UNI-PAC method to ground-based observations.

\section{Conclusions}

We have presented the principle of a pre-optics concept for precise wavefront aberration reduction in front of a coronagraph in a terrestrial-planet-finding telescope. It consists of a combination of an unbalanced nulling interferometer and a two-deformable mirror AO system with a conventional pupil plane wavefront sensor, where it does not meet the low intensity problem for wavefront measurements. This method reduces both the source intensity as a nulling coronagraph and the (speckle halo) noise intensity produced by wavefront aberrations.

The wavefront magnification phenomenon at the unbalanced nulling interference makes it possible to correct the wavefront precisely beyond the capabilities of employed AO systems with non-common path errors. By using the UNI-PAC, the specification of all the coronagraph optics can be relaxed: this is probably a key step in developing a cost-effective exo-planet detection system, although the performance of the entire system should be investigated.

A candidate instrument where the UNI-PAC system can be used would be a modified Michelson interferometer in a collimated beam of an off-axis telescope (Shao et al. 2004). We also expect the present concept to be applicable to several other coronagraphic concepts instead of the UNI stage by a Michelson beam combiner, i.e. with more general contrast-reduction setups (see for example Abe et al. 2006), but we leave this discussion to a future study.

Some other considerations, such as achromaticity, resolved star nulling, planet image quality, and AO performance limitations should be investigated.

Acknowledgements. L.A. is supported by Grant-in-Aid (Nos. 160772048007 and 160871018002) from the Ministry of Education, Culture, Sports, Science, and Technology (MEXT) of Japan. J.N. and N.M. are supported by Grant-in-Aid (No. 19360036 and the Priority Areas "Development of Extra-solar Planetary Science") from the MEXT. The authors are grateful to Profs. M. Yoshizawa and M. Tamura for their constant encouragements with this work.

\section{Appendix A: Wavefront aberration expressions}

The complex amplitude of an electric field in a pupil plane with a "classical" wavefront phase aberration $\theta(x, y)$ and an amplitude aberration $\alpha(x, y)$ can be written as

$E(x, y)=E^{0}(1+\alpha(x, y)) \mathrm{e}^{\mathrm{i} \theta(x, y)}$.

Here $E^{0}$ is the unaberrated amplitude (usually assumed to be a real number), and the effect of the aberrations can be defined as a deviation $\varepsilon$ between $E$ and $E^{0}$ noted,

$\varepsilon(x, y)=E(x, y)-E^{0}$,

which is the complex aberration component in Eq. (1), at the beginning of the formalism in Sect. 3. Relationships between $\varepsilon$

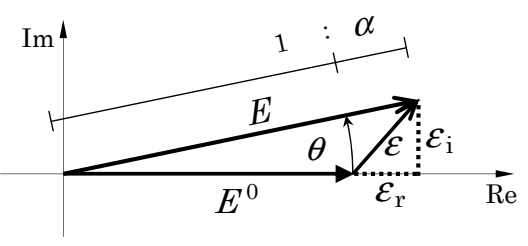

Fig. A.1. Correspondence of the electric field definitions.

and the "classical" wavefront aberrations can be seen in Fig. A.1, written as

$$
\left\{\begin{array}{l}
\tan \theta(x, y)=\frac{\varepsilon_{\mathrm{i}} / E^{0}}{1+\varepsilon_{\mathrm{r}} / E^{0}} \\
\alpha(x, y)=\sqrt{\left(1+\varepsilon_{\mathrm{r}} / E^{0}\right)^{2}+\left(\varepsilon_{\mathrm{i}} / E^{0}\right)^{2}}-1,
\end{array}\right.
$$

and

$$
\left\{\begin{array}{l}
\varepsilon_{\mathrm{i}} / E^{0}=(1+\alpha(x, y)) \sin \theta(x, y) \\
\varepsilon_{\mathrm{r}} / E^{0}=(1+\alpha(x, y)) \cos \theta(x, y)-1 \\
\varepsilon(x, y)=\varepsilon_{\mathrm{r}}+\mathrm{i} \varepsilon_{\mathrm{i}}
\end{array}\right.
$$

where $i$ is the imaginary unit, $\varepsilon_{\mathrm{r}}$ and $\varepsilon_{\mathrm{i}}$ are real numbers, which indicate the real and the imaginary part of $\varepsilon(x, y)$, respectively, and where $(x, y)$ is not indicated for simplicity.

$E^{0}$ can be defined as a spatial ensemble average of $E$ within the pupil plane, $E^{0}=\langle E(x, y)\rangle$ so that the aberration component has a zero mean, $\langle\varepsilon(x, y)\rangle=0$, whereas $\langle\alpha(x, y)\rangle$ and $\langle\theta(x, y)\rangle$ are not always necessarily zero. Under small wavefront aberration conditions $\left|\varepsilon / E^{0}\right| \ll 1$, as for the present method, approximations

$\left\{\begin{array}{l}\theta(x, y) \sim \varepsilon_{\mathrm{i}} / E^{0} \\ \alpha(x, y) \sim \varepsilon_{\mathrm{r}} / E^{0}\end{array}\right.$

can be used. Therefore, the normalized complex aberration, $\varepsilon(x, y) / E^{0}$ is a good indicator of the wavefront aberrations for our concept.

\section{Appendix B: Correlation between the two wavefront aberrations at the UNI stage}

At the UNI stage, if the complex aberrations of the two wavefronts correlate strongly and have almost the same amplitude, $\varepsilon_{\mathrm{A}}(x, y) \sim \varepsilon_{\mathrm{B}}(x, y)$, the difference between the two wavefronts at planes A and B tends to zero. Then, the aberration component of the UNI output becomes

$$
\left\{\begin{array}{l}
\varepsilon_{\mathrm{UNI}}(x, y) \sim g \varepsilon_{\mathrm{A}}(x, y) \\
\left\langle I_{\mathrm{UNI}}^{\varepsilon}(x, y)\right\rangle \sim g^{2}\left\langle I_{\mathrm{A}}^{\varepsilon}(x, y)\right\rangle,
\end{array}\right.
$$

instead of Eq. (11). This means that a simple nulling interference can reduce both the star intensity and the speckled halo. This constitutes a more suitable situation for achieving a deeper nulling without being concerned about aberration, but would be unrealistic.

If both wavefront aberrations are perfectly uncorrelated, the unbalanced nulling combination produces a larger complex aberration term. This is the "worst case" situation expressed by Eq. (11).

When the two wavefronts are taken at different positions in a telescope primary or a telescope-side AO system, they may partially correlate and the nulling combination provides results that are intermediate between those described above. 


\section{Appendix C: Planet intensity after the UNI stage}

We examine how an off-axis object (a planet) is affected by the UNI stage. We consider an interferometric beam combination scheme (two sub-pupils extracted from a monolithic telescope mirror, or using diluted apertures). During a "blind search" observation, we must consider the rotation of the nulling interferometer to explore the planet in every direction, which results in an averaging of the planet signal between the (pseudo-)nulled and constructive conditions of the interferometer for a given baseline $B$ (e.g. Bracewell 1978). The planet should also be sufficiently off-axis, by more than $\sim \lambda / B$, to prevent the planet staying in the central nulled region.

We introduce the complex amplitude of the planet $E_{\mathrm{A}}^{\mathrm{p}}\left(=E_{\mathrm{B}}^{\mathrm{p}}\right)$ and its intensity $I_{\mathrm{A}}^{\mathrm{p}}$, where we consider only the unaberrated amplitude and neglect the aberration term, since its intensity is small. The average of the planet intensities for the constructive, $\left|E_{\mathrm{A}}^{\mathrm{p}}-(1-g) E_{\mathrm{B}}^{\mathrm{p}}\right|^{2}$, and the destructive, $\left|E_{\mathrm{A}}^{\mathrm{p}}+(1-g) E_{\mathrm{B}}^{\mathrm{p}}\right|^{2}$, conditions after the UNI becomes

$I_{\mathrm{UNI}}^{\mathrm{p}}=\left(2-2 g+g^{2}\right) I_{\mathrm{A}}^{\mathrm{p}}$,

which should be used for calculation of the dynamic range improvement.

\section{References}

Abe, L., Murakami, N., Nishikawa, J., \& Tamura, M. 2006, A\&A, 451, 363 Aime, C., \& Soummer, R. 2004, Proc. SPIE, 5490, 456

Aime, C., \& Vakili, F. ed. 2006, Direct Imaging of Exoplanets (Cambridge: CUP), IAU Colloq. 200

Baba, N., Murakami, N., Ishigaki, T., \& Hashimoto, N. 2002, Optics Lett., 27, 1373

Balasubramanian, K., Hoppe, D. J., Halverson, P. G., et al. 2006, Direct Imaging of Exoplanets, ed. C. Aime, \& F. Vakili (Cambridge: CUP), IAU Colloq., 200, 405
Baudoz, P., Rabbia, Y., Gay, J., et al. 2000, A\&AS, 145, 341

Bordé, P. J., \& Traub, W. A. 2006, ApJ, 638, 488

Bracewell, R. N. 1978, Nature, 274, 780

Brown, R. A., Burrows, C. J., Casertano, S., et al. 2003, SPIE 4854, 95

Codona, J. L., \& Angel, R. 2004, ApJ, 604, 117

Coulter, D. R. 2005, Proc. SPIE, 5905

Evans, J. W., Morzinski, K., Reza, L., et al. 2005, SPIE, 5905, 303

Fienup, J. R. 1982, Appl. Opt., 21, 2758

Galicher, R., Guyon, O., Otsubo, M., Suto, H., \& Ridgway, S. 2005, PASP, 117, 411

Gerchberg, R. W., \& Saxton, W. O., Optik, 35, 237

Give'on, A., Kasdin, N., J., \& Vandervei, R. J. 2006, Direct Imaging of Exoplanets, ed. C. Aime, \& F. Vakili (Cambridge: CUP), IAU Colloq. 200, 541

Guyon, O. 2006, Direct Imaging of Exoplanets, ed. C. Aime, \& F. Vakili (Cambridge: CUP), IAU Colloq. 200, 559

Guyon, O., Roddier, C., Graves, J.E., et al. 1999, PASP, 111, 1321

Kasdin, N. J., Belikov, R., Beall, J., et al. 2005, Proc. SPIE, 5905, 59050G-1

Kuchner, M. J., \& Traub, W. A. 2002, ApJ, 570, 900

Lowman, A. E., Trauger, J. T., Gordon, B., et al. 2004, Proc. SPIE, 5487, 1246

Makidon, R. B., Sivaramakrishnan, A., Soummer, R., et al. 2006, Direct Imaging of Exoplanets, ed. C. Aime, \& F. Vakili (Cambridge: CUP), IAU Colloq. 200, 603

Malbet, F., Yu, J. W., \& Shao, M. 1995, PASP, 107, 386

Mennesson, B., Léger, A., \& Olliver, M. 2005, Icarus, 178, 570

Nishikawa, J., \& Murakami, N. 2006, Direct Imaging of Exoplanets, ed. C. Aime, \& F. Vakili (Cambridge: CUP), 427, IAU Colloq. 200

Nihsikawa, J., Kotani, T., Murakami, N., et al. 2005, A\&A, 435, 379

Riaud, P., Boccaletti, A., Baudrand, J., \& Rouan, D. 2003, PASP, 115, 712

Roggenmann, M. C., \& Lee, D. J. 1998, Appl. Opt., 37, 4577

Serabyn, E., Bloemhof, E. E., Gappinger, R. O., et al. 2006, Direct Imaging of Exoplanets, ed. C. Aime, \& F. Vakili (Cambridge: CUP), 477, IAU Colloq., 200

Shao, M., Wallace, J. K., Levine, B. M., \& Liu, D. T. 2004, Proc. SPIE, 5487, 1296

Tamura, M., Hodapp, K., Takami, H., et al. 2006, SPIE 6269, 62690V

Tavrov, A. V., Otani, Y., Kurokawa, T., \& Takeda, M. 2005, Proc. SPIE, 5905, 398

Tolls, V., Aziz, M., Gonsalves, R. A., et al. 2005, Proc. SPIE, 5905, 59051M-1

Zernike, F. 1934, MNRAS, 94, 377 